\title{
Gas-phase organics in environmental tobacco smoke: \\ 2. Exposure-relevant emission factors and indirect exposures from habitual smoking
}

Brett C. Singer ${ }^{1, *}$, Alfred T. Hodgson ${ }^{1}$, AND William W NAZAROFF ${ }^{1,2}$

${ }^{1}$ Environmental Energy Technologies Division, E. O. Lawrence Berkeley National Laboratory, Berkeley, CA 94720 USA

${ }^{2}$ Department of Civil and Environmental Engineering, University of California, Berkeley, CA 94720-1710 USA

\begin{abstract}
Sorption of emitted gas-phase organic compounds onto material surfaces affects environmental tobacco smoke (ETS) composition and exposures indoors. We have introduced a new metric, the exposure relevant emission factor (EREF) that accounts for sorptive uptake and reemission to give the mass of individual ETS constituents available for exposure over a day in which smoking occurs. This paper describes month-long experiments to investigate sorption effects on EREFs and potential ETS exposures under habitual smoking conditions. Cigarettes were smoked in a 50$\mathrm{m}^{3}$ furnished room over a 3-h period 6-7 days per week, with continuous ventilation at 0.3, 0.6, or $2.1 \mathrm{~h}^{-1}$. Organic gas concentrations were measured every few days over 4-h "smoking”, 10-h "post-smoking” and 10-h "background” periods. Concentration patterns of volatile ETS components including 1,3-butadiene, benzene and acrolein were similar to those calculated for a theoretical non-sorbing tracer, indicating limited sorption. Concentrations of ETS tracers, e.g., 3ethenylpyridine (3-EP) and nicotine, and lower volatility toxic air contaminants including phenol, cresols, and naphthalene increased as experiments progressed, indicating mass accumulation on surfaces and higher desorption rates. Daily patterns stabilized after week 2, yielding a steady daily cycle of ETS concentrations associated with habitual smoking. EREFs for
\end{abstract}

\footnotetext{
* Phone: 510-486-4779; Fax: 510-486-5892; E-mail: BCSinger@lbl.gov
} 
sorbing compounds were higher under steady-cycle versus single-day smoking conditions by 50\% for 3-EP, and by 2-3 times for nicotine, phenol, cresols, naphthalene, and methylnaphthalenes. Our results provide relevant information about potential indirect exposures from residual ETS (nonsmoker enters room shortly after smoker finishes) and from reemission, and their importance relative to direct exposures (nonsmoker present during smoking). Under the conditions examined, indirect exposures accounted for a larger fraction of total potential exposures for sorbing versus non-sorbing compounds, and at lower versus higher ventilation rates. Increasing ventilation can reduce indirect exposures to very low levels for non-sorbing ETS components, but indirect routes accounted for $~ 50 \%$ of potential nicotine exposures during non-smoking periods at all ventilation rates.

\section{Key Words}

Environmental tobacco smoke; toxic air contaminants; hazardous air pollutants; ETS tracers; nicotine; sorption; emission factors; exposure assessment.

\section{Introduction}

Environmental tobacco smoke (ETS) is a dynamic mixture of particulate and gas-phase compounds including many U.S. federally regulated hazardous air pollutants and California state-regulated toxic air contaminants (HAPs/TACs) (CARB, 2003). Regular exposure to ETS increases the risk of various health problems, including asthma, heart disease, and lung cancer (National Cancer Institute, 1999; National Research Council, 1986).

Indoor concentrations of ETS depend primarily on smoking frequency, dilution volume, and ventilation rate. Individual components of ETS are released in relatively constant proportion, but their concentrations indoors are variably influenced by transformation and removal processes. The most important differentiating process for many ETS gases is sorption to material surfaces. Adsorption can reduce concentrations of heavily-sorbing compounds relative to non- or lightly-sorbing compounds during and shortly after smoking. Subsequent desorption (reemission) can cause exposures to sorbing ETS compounds long after smoking has stopped. The initial 
reduction and later enhancement of exposure depend on sorptive interactions between ETS gases and all indoor surfaces. These in turn depend on factors such as the physical-chemical properties of the sorbing gases and the types and quantities of materials present in an interior space.

Exposures to a range of ETS constituents can be estimated by combining emission factors measured in controlled laboratory experiments with field measurements of exposure to one or more ETS-specific “tracer” compounds, the most common of which is nicotine (Hodgson et al., 1996). Martin et al. (1997) and Daisey et al. (1998) derived per-cigarette emission factors for a range of ETS components based on measurements in unventilated stainless steel test chambers. These data provide insight into ETS composition in a well-controlled setting, but may not be directly applicable to ordinary indoor environments. One approach to using such data is to account for sorption in the test chamber to calculate the total mass of each compound emitted from the burning end of the cigarette (i.e., sidestream smoke emissions). Theoretically, one could then account for sorption of individual compounds on all materials in an environment of interest (e.g., through modeling) and use this information to predict nonsmoker exposure to various ETS components. While sorption rate data are available for several combinations of ETS constituents and indoor materials (Van Loy et al., 2001; Won et al., 2001), data are unavailable for many others. Also, the scaling of individual material results to predict concentrations in a full-scale space with an array of surfaces has yet to be successfully demonstrated.

We previously presented an alternative approach in which exposure relevant emission factors (EREFs) were measured for 26 organic gases and ETS tracers in a realistically constructed, furnished and ventilated room-sized test chamber (Singer et al., 2002). EREFs were measured for three smoking levels $\left(5,10,20 \mathrm{cig}^{-1}\right)$, three ventilation rates $\left(0.3,0.6,2 \mathrm{~h}^{-1}\right)$ and three furnishing conditions (wallboard with aluminum flooring, wallboard with carpet, full furnishings). Single-day EREFs include mass that is initially removed from the air through sorptive uptake but is relevant to exposures because it is reemitted within a 24-h period. The sorbing portion of the emitted mass is often excluded from conventional emission factor measurements and calculations. Calculated single-day EREFs were unaffected by the number of 
cigarettes, suggesting that sorption was linearly related to gas-phase concentration. EREFs varied little with furnishings or ventilation for many volatile HAPs/TACs including 1,3-butadiene, acrolein, and benzene. By contrast, EREFs varied by factors of 2-10 for compounds that sorbed to material surfaces; these compounds included naphthalene, methylnaphthalenes, phenol, cresols, nicotine and other ETS-specific tracers. Ongoing reemission was confirmed by detection of these compounds in the gas-phase for weeks to months after the single day of smoking. Since EREFs represent the emitted mass available for exposure over a single day in which smoking occurs, they may be used with indoor air quality models to estimate daily ETS exposures for varied smoking rates and house parameters.

Questions remain from our earlier work. In most indoor environments in which smoking occurs, the smoking is habitual and cyclic. Does the accumulation of sorbing species over time alter the sorptive interactions in such a manner as to affect the composition of ETS? Also, what roles do sorptive interactions play in altering the time-pattern of exposure in indoor environments in which repeated cycles of smoking occur? This paper reports on a series of experiments designed to investigate these questions.

\section{Experimental Methods}

The dynamics of ETS constituents were studied at three air-exchange rates in a simulated indoor environment containing furniture and other typical residential materials that provided appropriate sites for sorption. Cigarettes were machine-smoked within a 3-h interval each day, intended to represent a working adult smoking at home each evening. Gas-phase organic compounds were measured during three daily periods corresponding to active smoking, postsmoking, and daily background conditions. The protocols generally followed those used previously (Singer et al., 2002). Experimental details are summarized in Table 1.

\subsection{Chamber}

The ceiling and walls of the $50-\mathrm{m}^{3}$ chamber were finished with $64.2 \mathrm{~m}^{2}$ of painted gypsum wallboard. The 20.4- $\mathrm{m}^{2}$ floor was covered with unpadded nylon carpet. Additional 
surface area was provided by several pieces of solid wood and veneer furniture $\left(18.7 \mathrm{~m}^{2}\right)$ and four upholstered chairs with polyester fabric $\left(13.9 \mathrm{~m}^{2}\right)$. Two sections of pleated cotton draperies covered $10.5 \mathrm{~m}^{2}$ of wall space. All materials were exposed previously to tobacco smoke, but carry-over effects were reduced by spacing experiments several months apart. Pre-experiment background samples contained low, but measurable concentrations of several target compounds.

The chamber was housed within a small building. All ventilation air was supplied to the chamber by drawing outdoor air though activated carbon to remove organic gases. Ventilation rates of approximately $0.3,0.6$ and $2.1 \mathrm{~h}^{-1}$ were selected to represent low, moderate and high average values for typical residences. The actual ventilation rates shown in Table 1 were measured by first-order decay of injected $\mathrm{SF}_{6}$ during multiple days of each experiment. Chamber air was circulated using four small (10 cm diameter) axial fans placed $\sim 1 \mathrm{~m}$ from the corners, alternately at $1 / 3$ and 2/3 of the room height.

Chamber air temperature was controlled at $20-23{ }^{\circ} \mathrm{C}$ during all experiments by regulating the building temperature. Relative humidity followed a diurnal pattern and was generally lower and more variable in experiments 2 and 4, which were conducted during winter. In experiment 3, RH gradually increased from about $30 \%$ on day 4 to about $40 \%$ on day 8 then mostly remained in the range of $40-50 \%$.

\subsection{ETS generation}

ETS was simulated using the sidestream emissions of a leading brand of filter cigarettes. Cigarettes were purchased by the carton from local retailers and smoked without special preparation. Results presented by Daisey et al. (1998) showed that ETS emissions of VOC were relatively consistent among the leading brands tested. The cigarettes used in the current study were previously identified as Brand A (ibid). The smoking machine consisted of a single port puffer (ADL/II smoking system, Arthur D. Little, Inc.) attached to a twelve-port carousel, positioned $>1 \mathrm{~m}$ from the nearest wall. The puffer drew one $35-\mathrm{cm}^{3}, 2-\mathrm{s}$ puff every minute and exhausted the mainstream smoke from the chamber. Smoking started at the same time on each 
day of a given experiment, but two different smoking schedules were employed. When gas samples were collected during each daily period (full sampling day), cigarettes were smoked at equal intervals over a 3-h period. On other days, cigarettes were smoked one after another and gas samples were collected only during the background period. Smoking occurred 6 days per week in experiments 1 and 2 and every day during the others. The smoking rate was either 5 or 10 cigarettes per day.

\subsection{Gas-phase organic compound sampling and analysis}

Target compounds shown in Table 2 include HAPs/TACs, ETS-specific tracers, and other major components of ETS. They range from very volatile organic compounds (VVOCs) with vapor pressures of $>0.3 \mathrm{~atm}$ (e.g., 1,3-butadiene and acrolein) to semivolatile organic compounds (SVOCs) having vapor pressures of $<10^{-4}$ atm (e.g., methylnaphthalenes and nicotine). The compounds in Table 2 are grouped by functionality and roughly ordered by vapor pressure.

Air samples were collected primarily on Tenax-TA ${ }^{\mathrm{TM}}$ sorbent tubes (P/N CP-16251; Varian, Inc.) modified by substituting a 15-mm section of Carbosieve S-III 60/80 mesh (P/N 10184, Supelco Inc) for the Tenax-TA ${ }^{\mathrm{TM}}$ at the outlet end. Sorbent tubes were attached to holders constructed from stainless steel tubing and inserted through ports in the chamber wall so that air was drawn directly into the tubes from the room, 0.4-0.6 $\mathrm{m}$ away from the wall. Air was pulled through the sorbent tubes using peristaltic pumps located outside the chamber. Pump flow rates were measured throughout each experiment. The large majority of samples throughout experiments 2-4 were collected at approximately $5 \mathrm{cc} / \mathrm{min}$, yielding volumes up to $3 \mathrm{~L}$. A subset of daily background samples collected at $\sim 16 \mathrm{cc} / \mathrm{min}$ onto Tenax tubes during experiment 4 yielded concentrations consistent with lower volume samples.

Sorbent tube samples were quantitatively analyzed by thermal desorption-gas chromatography/mass spectrometry (TD-GC/MS) generally following U.S. EPA Method TO-1 (U.S. EPA, 1984). Chemical analysis methods were similar to those reported by Daisey et al. 
(1998) except that samples were thermally desorbed and concentrated on a cryogenic inletting system (Model CP-4020 TCT; Varian, Inc.). The system was fitted with a Tenax-packed trap (P/N CP-16425; Varian, Inc.) to avoid loss of the most volatile compounds. Desorption temperature was set to $235^{\circ} \mathrm{C}$ for $6.5 \mathrm{~min}$. The cryogenic trap was held at $-100{ }^{\circ} \mathrm{C}$ and then heated to $235^{\circ} \mathrm{C}$ for injection. Multi-point calibrations were referenced to an internal standard of 1-bromo-4-fluorobenzene.

Air samples were collected at $\sim 1 \mathrm{~L} \mathrm{~min}^{-1}$ onto treated silica-gel cartridges (P/N WAT047205, Waters Corp.) for the measurement of formaldehyde and acetaldehyde. Each cartridge was extracted with $2 \mathrm{~mL}$ of acetonitrile and the extracts were analyzed by highperformance liquid chromatography (HPLC) with a diode array detector at a wavelength of 365 nm (ASTM, 1997). Extract concentrations were determined based on multipoint calibrations of external standard mixtures.

\subsection{Sampling protocol}

Concentrations of gas-phase organic compounds were measured during three periods representing conditions during, shortly after, and long after daily smoking. A 4-h "smoking” period encompassed the 3-h interval of actual smoking plus one additional hour to reduce the effect of recent emissions on the next period. The 10-h “post-smoking” period represents overnight for an evening smoking scenario. The diurnal cycle was completed with a 10-h "background" sample representing the morning and afternoon following an evening smoking event. Experiment 1 used a slightly different scheme, with an 8-h post-smoking period and a 12h background period. Sorbent tube samples were collected during the background period before the first day of smoking and during each period of full sampling days (Table 1). Complete sets of aldehyde samples were collected during experiments 1 and 2. Aldehydes were not measured during experiment 3 (same conditions as experiment 1). In experiment 4, valid aldehyde measurements were obtained for days 1, 4, 6, 8, 11, and 14, but not for the pre-experiment background samples; therefore steady cycle EREFs were not calculated for aldehydes during this 
experiment. Acetaldehyde concentrations were lower than formaldehyde in pre-experiment and daily background periods and much higher during experiments, yielding more certain EREFs for acetaldehyde than formaldehyde.

Duplicate organic gas samples were collected during many periods and concentrations calculated from co-located samples agreed within $\pm 10 \%$ in almost all cases.

Compound concentrations exceeded lower limits of quantitation for all smoking and most post-smoking periods, except for 1,3-butadiene through butanone (Table 2) during experiment 3 at the high ventilation rate. In this experiment, daily background concentrations of these same compounds were indistinguishable from quantitation limits or pre-experiment background concentrations.

\subsection{Calculation of emission factors}

Organic compound concentrations during all experimental sampling periods $\left(\mathrm{C}_{\mathrm{pd}}\right)$ were adjusted by subtracting concentrations measured before the start of each experiment $\left(\mathrm{C}_{\mathrm{pre}}\right)$. This chamber background adjustment primarily accounts for the small quantity of outdoor organic gases (mostly aromatics) that passed through the activated carbon bed on the ventilation supply air and for any emissions by room materials (e.g. formaldehyde). These net concentrations ( $\mu$ g $\mathrm{m}^{-3}$ ) were multiplied by the measured air-exchange rate $\lambda\left(\mathrm{h}^{-1}\right)$, room volume $\mathrm{V}\left(50 \mathrm{~m}^{3}\right)$, and period duration $\Delta \mathrm{t}(\mathrm{h})$ to calculate the mass $(\mu \mathrm{g})$ of each constituent removed by means of ventilation from the chamber during each period. EREFs ( $\left.\mu \mathrm{cig}^{-1}\right)$ were calculated by dividing the total mass removed over the three periods of a full daily cycle by the number of cigarettes smoked, $\mathrm{N}_{\text {cig. }}$ This calculation, summarized by Equation 1 below, was applied for each target compound during each sampling day for three periods (pd = smoke, post-smoke, background).

$$
E R E F=\frac{\sum_{p d}\left(C_{p d}-C_{p r e}\right) \lambda V \Delta t}{N_{c i g}}
$$


Equivalently, the total mass could be calculated as the product of the time-averaged concentration and the volume of air moving through the room during each 24-h cycle. The difference between this value and the total mass emitted as sidestream smoke is the net amount that remains sorbed to surfaces after one day. Steady-cycle EREFs were calculated from the average concentrations measured on all full sampling days after the first two weeks of each experiment, whereas initial EREFs were calculated from concentrations averaged over the first two full sampling days (Table 1).

The chamber background adjustment procedure described above had little effect on steady-cycle EREFs for most compounds in experiments 2 and 4 , at 0.6 and $0.3 \mathrm{~h}^{-1}$, since preexperiment concentrations were much lower than even the daily background concentrations measured by the middle of these experiments. However, at the highest ventilation rate daily background period concentrations of the least volatile compounds were only a few times higher than pre-experiment concentrations. Since the only source for these compounds in the room was prior ETS emissions, we assumed that the observed steady cycle concentrations would have been reached regardless of their pre-experiment levels. Therefore, for experiment 3, no background adjustments were made for compounds below naphthalene in Table 2.

Uncertainty of individual EREFs was estimated using the compound specific coefficients of variation (CVs) provided in Table 2. For most compounds, CVs relevant to the experimental approach used in this study were determined and presented in our previous study (Singer et al., 2002). Method CVs for formaldehyde and acetaldehyde were calculated from the variability of concentrations measured during each sample period over multiple days of an experiment, with results averaged together for the two experiments with sufficient data $(n=6)$. As a check for all compounds, EREFs were calculated on individual sampling days during the final two weeks of each experiment, i.e. during steady-cycle conditions. The variability of these single-day EREFs was generally consistent with or smaller than the CVs shown in Table 2. 


\section{Results and discussion}

\subsection{Temporal trends in ETS organic gas concentrations}

The experiments were designed to study the effect of sorption processes on ETS composition over the course of a single day and over several weeks of daily smoking. Figure 1 illustrates the temporal patterns for toluene, 3-ethenylpyridine (3-EP), and 2-methylnaphthalene, compounds that respectively exhibited little, moderate, and substantial levels of sorption in the simulated indoor environment.

The daily pattern was driven primarily by the smoking schedule and ventilation rate of each experiment, but sorption processes played a role for many compounds. As illustrated in Figure 1, concentrations of all compounds were highest during the 4-h smoking period, substantially lower during the 10-h post-smoking period, and lowest during the 10-h daily background period. Diurnal variability increased with ventilation rate for all compounds, but the variability was reduced when sorption occurred. For example, toluene concentrations were approximately 3 times higher during the smoking period than during the post-smoking period at the low ventilation rate $\left(0.3 \mathrm{~h}^{-1}\right), 5$ times higher at $0.6 \mathrm{~h}^{-1}$ and 14 times higher at $2.1 \mathrm{~h}^{-1}$. By contrast, the ratios of smoking to post-smoking concentrations for 3-EP were 2.3, 2.7, and 6, respectively, at low, medium and high ventilation rates. The ratios were even lower for 2methylnaphthalene: $1.8,2.2$, and 4 , respectively.

Figure 1 also illustrates trends in the daily pattern of ETS concentrations over the course of each experiment. The trend varied for each daily period, by ventilation rate and according to the sorption properties of the compounds. Concentrations of the most volatile compounds (those listed above benzene in Table 2) varied from day to day, but showed no consistent trend with time as each experiment progressed. In some cases, small increases appeared over time for the aromatic compounds (benzene through styrene in Table 2). But as shown for toluene in Figure 1, differences between the concentrations on the first few days versus later days were often of the same magnitude as the variability during the later days of each experiment. Concentrations of less volatile compounds and ETS tracers (naphthalene and below in Table 2) increased markedly 
from day-to-day during the early phases of the low and moderate ventilation rate experiments, as shown for 3-EP and 2-methylnaphthalene in Figure 1. Smaller daily increases were observed for most of these compounds at the high ventilation rate. Day-to-day increases were greater at lower ventilation rates and at the times least influenced by fresh emissions, i.e., during the postsmoking and background periods.

The plots in Figure 1 also show that a steady-cycle of daily concentrations appears to be achieved on a time scale of 1-2 weeks for all compounds at the ventilation rates studied. We characterize as steady cycle the period after which concentrations vary from day to day without a directional trend. For example, 3-EP concentrations followed a steady daily cycle after about a week at 0.3 and $0.6 \mathrm{~h}^{-1}$, and after a few days at $2.1 \mathrm{~h}^{-1}$. Naphthalene, pyridine, pyrrole and the picoline isomers behaved similarly to 3-EP. Concentrations of methylnaphthalenes, phenol, cresols, nicotine and myosmine rose sharply over the first two weeks at 0.3 and $0.6 \mathrm{~h}^{-1}$, and over $\sim 10$ days during the experiment at $2.1 \mathrm{~h}^{-1}$, as illustrated by 2-methylnaphthalene. The steadycycle patterns observed in our experiments are representative of conditions resulting from regular smoking on the time scale of weeks to months. We note that patterns could differ somewhat over the multi-year time scales over which habitual smoking occurs in some residences.

The progression of daily concentrations is consistent with at least a partially reversible sorption process in which mass accumulates on surfaces during the early days of an experiment, followed by increased desorption and higher concentrations on later days. Desorption had the largest effect on concentrations during daily post-smoking and background periods, i.e. when there were lower levels of residual mass remaining in chamber air from the last emission event. Concentrations at steady-cycle conditions reflect a dynamic balance among emission, sorptive uptake and reemission processes.

The non-smoking days of the moderate ventilation experiment provide insight into the desorption process. 2- Methylnaphthalene background period concentrations dipped only slightly on the non-smoking days 7 and 21, suggesting that the mass desorbed over one day was small compared to the total mass sorbed (if a substantial fraction of the mass desorbed in one day, the 
rate would drop accordingly). By contrast, background concentrations of 3-EP were much lower on these days than on adjacent days. Without the contribution of newly sorbed mass from fresh emissions, the sink of reversibly sorbed 3-EP mass was noticeably depleted within a day.

\subsection{Exposure-relevant emission factors}

The elevated organic gas concentrations that result from decreased sorption and increased reemission under daily smoking conditions have important implications for ETS exposures. We accounted for these effects by calculating EREFs for each compound on each full sampling day of each experiment. Results are presented in Figure 2 for benzene, naphthalene, and nicotine. Consistent with the discussion above, Figure 2 shows that EREFs stabilized after the first 1-2 weeks of each experiment. EREFs calculated from concentrations averaged over the last two weeks of each experiment are shown in Table 2. EREFs for high volatility compounds, i.e. formaldehyde through acetonitrile, did not increase during the experiments. EREFs for the aromatic compounds increased slightly with time in some, but not all cases. Averaged across the three ventilation rate experiments, EREFs during the third and fourth weeks were about 15-30\% higher than those measured during the first 3 smoking days for the aromatic compounds and for several ETS tracers (pyridine through pyrrole). The largest increases were observed for the less volatile compounds and ETS tracers, as shown for naphthalene and nicotine in Figure 2. Steadycycle EREFs for naphthalene, methylnaphthalenes, phenol, cresols, nicotine, and myosmine were about 65-100\% higher, on average, than EREFs measured during the initial two or three days of each experiment. In between these groups was 3-EP, with steady-cycle EREFs about 45\% higher, on average, than early day EREFs.

Steady cycle EREFs shown for the very volatile and aromatic compounds in Table 2 (i.e. 1,3-butadiene through 1,2,4-trimethylbenzene) were on average about 10-45\% higher than the single-day EREFs reported previously for the same ventilation and furnishing conditions (Singer et al., 2002). Differences of this magnitude were observed also for the most volatile ETS tracers (pyridine and picoline isomers). Steady cycle EREFs for pyrrole and 3-EP were about 60-70\% 
higher than those measured from a single day of smoking, while steady cycle EREFs for the remaining organic compounds (naphthalene through m-cresol) and the ETS tracers nicotine and myosmine were about 2-3 times higher than single-day EREFs measured under similar ventilation and furnishing conditions.

For almost all compounds that were substantially affected by sorption processes (i.e., all compounds below naphthalene in Table 2), steady cycle EREFs under fully furnished conditions were similar to single-day EREFs measured when the chamber contained only wallboard or wallboard and carpet (Singer et al., 2002). At the lowest ventilation rate, steady cycle EREFs for naphthalene, methylnaphthalenes, phenol and cresols, exceeded the single-day EREFs measured under wallboard only conditions at the same ventilation rate. These results show that reemission strongly influences 24-h average exposures when smoking occurs regularly over a time scale of one month. At least some of the mass lost to sorption on a single day of smoking appears to desorb over many days. When smoking occurs on a regular basis, the sorbed mass concentration increases, causing an increase in the desorption rate. Higher desorption rates produce higher concentrations and exposures under habitual smoking conditions.

Sorption processes affect potential exposures under habitual smoking conditions, as shown by the dependence of steady cycle EREFs on ventilation rate for several compounds (Table 2). But the effects generally were not as large for steady cycle conditions as they were for single-day EREFs. The decrease in steady cycle EREFs with decreasing ventilation rate suggests that airborne mass continued to be lost to sorptive uptake on a daily basis. This observation is consistent with diffusion into or through the materials present in the room, and also with a model of partially irreversible nicotine sorption proposed by Piade et al. (1999).

The relationship among steady-cycle EREFs measured for each compound across experiments informs about the potential for controlling ETS exposures by increasing ventilation. For compounds having similar EREFs across experiments (i.e., non- or lightly-sorbing gases), increasing ventilation will cause a proportional drop in exposures since the emitted mass will be diluted more rapidly. By contrast, Table 2 shows that the potential for exposure reduction using 
ventilation varies for sorbing compounds. The similarity in methylnaphthalene, phenol, and cresol EREFs at $0.3 \mathrm{~h}^{-1}$ versus $0.6 \mathrm{~h}^{-1}$ indicates that (for equivalent smoking rates) this ventilation increase should reduce concentrations and exposures by about a factor of 2 . However, the same ventilation increase would have essentially no effect on daily average nicotine concentrations since the EREF for nicotine at $0.6 \mathrm{~h}^{-1}\left(1660 \mu \mathrm{g} \mathrm{cig}^{-1}\right)$ is twice the EREF measured at $0.3 \mathrm{~h}^{-1}(820$ $\left.\mu \mathrm{g} \mathrm{cig}^{-1}\right)$.

Exposures to non-sorbing compounds will be reduced in proportion to ventilation increases over time scales that are long relative to smoking and ventilation, e.g. over a 24-h period. But the benefit of increasing ventilation may not scale directly for exposures that occur during and shortly after smoking. To illustrate this, we used equation 2 below to calculate mean concentrations during the nominal $4 \mathrm{~h}$ smoking period for a theoretical non-sorbing tracer and for toluene at air exchange rates of 0.3 and $2.1 \mathrm{~h}^{-1}$. Eq 2 superimposes contributions from the first order decay of emissions from 5 cigarettes smoked at times $t_{\mathrm{i}}$. The calculated ratio of smoking period concentrations at 0.3 and $2.1 \mathrm{~h}^{-1}$ is $3.9\left(\mathrm{t}_{\mathrm{i}}=0,0.6,1.2,1.8,2.4 \mathrm{~h}\right)$ for a non-sorbing tracer. Using the reported EREFs for toluene at these two air exchange rates yields a ratio of 2.8; this is slightly higher than the ratio of toluene concentrations measured during the smoking periods at the specified air exchange rates (as shown in Fig 1). Thus, an approximate sevenfold increase in ventilation rate reduced toluene concentrations by only about three times during the smoking period.

$\bar{C}_{0-4}=\sum_{i=1}^{5 c i g s} \frac{\left(\frac{E R E F}{V}\right)\left[\frac{\exp (-\lambda t)}{-\lambda}\right]_{0}^{4 h-t_{i}}\left(4 h-t_{i}\right)}{4 h}$ 


\subsection{Indirect exposures to ETS organic gases and tracers}

The design of these experiments allows us to investigate the importance of potential exposures through both direct and indirect pathways. Direct exposures occur when a nonsmoker occupies the same room as a smoker during active smoking. Indirect exposures can result from two mechanisms in a room where smoking occurs. Nonsmokers can enter the room after smoking has ceased but still be exposed to residual airborne ETS, i.e. to mass in the air that has not yet been removed by ventilation. Exposure may also occur from the reemission of ETS compounds that were previously sorbed to surfaces in the room. The first pathway is relevant to all ETS components while the second occurs only for compounds that reversibly sorb to indoor surfaces.

The potential importance of indirect exposures is demonstrated by the concentration data resolved by daily period. Steady-cycle concentrations of 2-methylnaphthalene during the 10-h post-smoking and 10-h background periods were $40-50 \%$ as high as the concentrations observed during the 4-h smoking period at low ventilation, and 30-45\% as high as those measured during the smoking period at moderate ventilation (Figure 1). These effects were less pronounced for moderately sorbing compounds such as 3-EP, but post-smoking and background period concentrations still reached levels that were a substantial fraction of those observed during smoking periods.

Gas-phase concentration data were used to calculate the total "potential” exposure during each daily period. Total potential exposure is computed assuming occupancy of an exposed individual $100 \%$ of the time, and thus accounts for the longer duration of post-smoking and daily background periods relative to the smoking period. Total potential exposures were apportioned into the three daily periods. Results are shown in Figure 3 for selected compounds. Included in this figure are results for a theoretical non-sorbing, non-reactive compound emitted on the actual smoking schedule. A daily concentration profile for the theoretical species was calculated by modeling the room as a well-mixed compartment and considering only the effects of emissions and removal by ventilation. 
Overall, the results indicate that ventilation rate is an important factor in determining the magnitude of indirect exposures for most compounds studied. At high ventilation rates, mass is quickly removed from the room and most of the potential hazard is limited to direct exposures during the smoking period. For example, at $2.1 \mathrm{~h}^{-1}$, more than $90 \%$ of daily exposure to formaldehyde, acetaldehyde, butadiene, acrolein, acrylonitrile, benzene, toluene, and other nonor lightly sorbing compounds occurred during the 4-h smoking period. Potential indirect exposures to these compounds were greater at lower ventilation rates, primarily as a result of residual ETS mass that remained in room air during the post-smoking period.

The potential timing of exposures to sorbing compounds, represented by naphthalene, ocresol, 3-EP, and nicotine in Figure 3, was controlled by both ventilation and sorption. At each ventilation rate, sorption shifts potential exposures away from the smoking period, resulting in fractionally higher indirect exposures. Even at the highest ventilation rate $\left(2.1 \mathrm{~h}^{-1}\right)$, only 37-53\% of potential exposures to naphthalene, methylnaphthalenes, phenol, and cresols occurred during the daily smoking period. Direct routes accounted for only $36-40 \%$ of potential daily exposure to these compounds at $0.6 \mathrm{~h}^{-1}$, and only $29-33 \%$ at $0.3 \mathrm{~h}^{-1}$. Stated conversely, indirect exposure routes accounted for at least $47-71 \%$ of potential daily exposures to these compounds at the conditions studied; we use the qualifier "at least” because the smoking period includes one hour after the last cigarette is smoked, i.e. a period of potential indirect exposure. At low and moderate ventilation rates, approximately $10-30 \%$ of potential exposure to these compounds occurred during the daily background period, i.e. 11-21 h after the last cigarette was smoked each day. These results indicate that any daily occupancy in a room where regular smoking occurs likely leads to indirect exposures to sorbing organic compounds through reemission from indoor surfaces. The sharp discrepancies between results for sorbing compounds versus the theoretical non-sorbing compound indicate that sorption must be considered to understand the dynamics of semivolatile ETS organic gases.

Figure 3 shows also that no single ETS tracer can be used to represent the dynamics of all ETS gases under all conditions. The distribution of 3-EP exposures during three daily periods 
was intermediate between the non-sorbing and sorbing organic compounds studied at the high ventilation rate, but behaved more similarly to the sorbing compounds at moderate and low ventilation rates. For nicotine and myosmine, the results were even more complex: the direct pathway accounted for about 50-55\% of total daily exposure to both compounds at all ventilation rates. These compounds behaved similarly to several of the sorbing HAPs/TACs at the high ventilation rate, but behavior differed at the lower ventilation rates.

Potential indirect exposures are shown on an absolute scale in Figure 4 for several representative compounds, including the volatile HAPs/TACs acrolein and benzene, the less volatile naphthalene, and the most commonly used gas-phase ETS tracers 3-EP and nicotine. The values shown were calculated using the measured post-smoking and background concentrations during weeks 3-4. The values for $0.6 \mathrm{~h}^{-1}$ were divided by two to account for the higher smoking rate in experiment $2\left(10\right.$ cig d $\left.^{-1}\right)$ compared with the other experiments $\left(5 \mathrm{cig} \mathrm{d}^{-1}\right)$. Figure 4 shows that absolute exposures to non-sorbing ETS constituents can be reduced to very low levels by increasing ventilation rates and by prohibiting smoking for at least an hour before a nonsmoker enters a room. For naphthalene and 3-EP, residual and reemission exposures can be reduced in approximate inverse proportion to ventilation. Nicotine exposures are less sensitive to ventilation and substantial exposures can occur even at high ventilation conditions. This finding demonstrates the importance of assessing the value and limitations of nicotine as a proxy for exposures to gas-phase components of ETS.

\section{Conclusions}

The results of this study indicate that nonsmoker exposure to volatile HAPs/TACs from ETS can be reduced to very low levels by the combined measures of increasing ventilation during smoking and prohibiting smoking when a nonsmoker is at home. However, exposure to lower volatility HAPs/TACs (e.g., cresols, naphthalene, polycyclic aromatic hydrocarbons), and to the ETS tracers nicotine and 3-EP, cannot be equivalently reduced with these measures. Nonsmokers who avoid direct exposure to ETS still may be exposed to moderate levels of 
nicotine, 3-EP, and lower volatility air toxics, even as exposures to ETS particles or volatile air toxics are reduced. One ramification is that an epidemiological study using nicotine or cotinine as the primary indicator of ETS exposure might classify these subjects as “exposed.” For a health outcome that is caused by exposure to ETS particles or non-sorbing gases, this classification would "dilute" the exposed group with subjects that were relatively unexposed to the ETS component(s) of interest. Conversely, there are circumstances in which nicotine or cotinine would be expected to underpredict exposure to particles or non-sorbing gases, such as when ETS exposure occurs in a nonsmoking room because of transport from a smoking environment. Compensating for such misclassification biases would improve the relative risk estimates for adverse health outcomes related to exposure to ETS particles or non-sorbing gases.

ETS exposure assessment is complicated both by the large number of individual toxic components and by the differential behavior of ETS toxics and tracers. Yet direct measurement of real world exposures to individual ETS components is costly and potentially confounded by non-ETS sources of the same compounds. Tracer techniques therefore will continue to play an important role in studying ETS health risks and evaluating the effectiveness of exposure controls. The data from this study provide information and insight into dynamic relationships among tracers and other ETS constituents in a realistic indoor environment. Application of our methods to other simulated and field settings will strengthen the empirical database. The next research priority is the development of a multicomponent sorption model that can reproduce empirical results accurately enough to allow for extrapolation and a more thorough examination of indirect exposures. Advancing our understanding of ETS dynamics indoors should lead to more efficient and effective interventions to reduce risk.

\section{Acknowledgements}

We gratefully acknowledge the contributions of the late Dr. Joan Daisey during the early phases of this work. We thank Toshifumi Hotchi, David Behnken and Karla Guevarra for their assistance with experimental, laboratory, and data handling tasks, and Melissa Lunden for 
reviewing the manuscript. This study was sponsored by the California Tobacco-Related Disease Research Program, Award 7RT-0099. Additional support was provided by the U.S. Department of Energy under Contract No. DE-AC03-76SF00098.

\section{References}

ASTM, 1997. Standard Test Method for Determination of Formaldehyde and Other Carbonyl Compounds in Air (Active Sampler Methodology). Method D5197-97, American Society for Testing and Materials, West Conshohocken, PA.

CARB, 2003. Toxic Air Contaminant Identification List. Up-to-date information available at http://www.arb.ca.gov/toxics/id.htm, Air Quality Measures Branch, California Air Resources Board, Sacramento, CA. Last accessed 13 March 2003.

Daisey, J.M., Mahanama, K.R.R., Hodgson, A.T., 1998. Toxic volatile organic compounds in simulated environmental tobacco smoke: Emission factors for exposure assessment. Journal of Exposure Analysis and Environmental Epidemiology 8, 313-334.

Hodgson, A.T., Daisey, J.M., Mahanama, K.R.R., Ten Brinke, J., Alevantis, L.E., 1996. Use of volatile tracers to determine the contribution of environmental tobacco smoke to concentrations of volatile organic compounds in smoking environments. Environment International 22, 295-307.

Martin, P., Heavner, D.L., Nelson, P.R., Maiolo, K.C., Risner, C.H., Simmons, P.S., Morgan, W.T., Ogden, M.W., 1997. Environmental tobacco smoke (ETS): A market cigarette study. Environment International 23, 75-90.

National Cancer Institute, 1999. Health Effects of Exposure to Environmental Tobacco Smoke: The Report of the California Environmental Protection Agency. Smoking and Tobacco Control Monograph No. 10. NIH Pub. No. 99-4645, U.S. Department of Health and Human Services, National Institutes of Health, National Cancer Institute, Bethesda, MD. 
National Research Council, 1986. Environmental Tobacco Smoke. Measuring Exposures and Assessing Health Effects. National Academy Press, Washington, D.C.

Piade, J.J., D’Andres, S., Sanders, E.B., 1999. Sorption phenomena of nicotine and ethenylpyridine vapors on different materials in a test chamber. Environmental Science \& Technology 33, 2046-2052, 1999.

Singer, B.C., Hodgson, A.T., Guevarra, K.S., Hawley, E.L., Nazaroff, W.W., 2002. Gas-phase organics in environmental tobacco smoke. 1. Effects of smoking rate, ventilation, and furnishing level on emission factors. Environmental Science \& Technology 36, 846-853.

U.S. EPA, 1984. Method TO-1, Revision 1.0: Method For The Determination Of Volatile Organic Compounds in Ambient Air Using Tenax ${ }^{\circledR}$ Adsorption and Gas Chromatography/Mass Spectrometry (GC/MS), Center for Environmental Research Information, Office of Research and Development, United States Environmental Protection Agency.

Van Loy, M. D., Riley, W.J., Daisey, J.M., Nazaroff, W.W., 2001. Dynamic behavior of semivolatile organic compounds in indoor air. 2. Nicotine and phenanthrene with carpet and wallboard. Environmental Science \& Technology 35, 560-567.

Won, D., Corsi, R.L., Rynes, M., 2001. Sorptive interactions between VOCs and indoor materials. Indoor Air 11, 246-256. 
Table 1. Summary of experiments.

\begin{tabular}{|c|c|c|c|c|c|c|c|c|}
\hline $\begin{array}{l}\text { Expt. } \\
\text { No. }\end{array}$ & $\begin{array}{l}\text { Vent. } \\
\text { rate }^{\mathrm{a}} \\
\left(\mathrm{h}^{-1}\right)\end{array}$ & $\begin{array}{l}\text { Smoke } \\
\text { rate } \\
\left(\text { cig d }^{-1}\right)\end{array}$ & $\begin{array}{l}\text { Smoke } \\
\text { pattern } \\
\left(\mathrm{d} \mathrm{wk}^{-1}\right)\end{array}$ & $\begin{array}{c}\text { Active } \\
\text { smoking } \\
\text { period }\end{array}$ & $\begin{array}{c}\text { Full sampling } \\
\text { days }\end{array}$ & $\begin{array}{l}\mathrm{RH} \\
(\%)\end{array}$ & Season & $\begin{array}{l}\text { Valid } \\
\text { data }^{c}\end{array}$ \\
\hline 1 & 2.09 & 10 & 6 & $\begin{array}{l}0900- \\
1200\end{array}$ & $\begin{array}{l}1,3,5,9,12 \\
16,19,23,34\end{array}$ & N/A & Summer & ALD \\
\hline 2 & 0.63 & 10 & 6 & $\begin{array}{l}1800- \\
2100\end{array}$ & $\begin{array}{l}1,3,6,10 \\
17,24,27\end{array}$ & $15-40$ & Winter & $\begin{array}{l}\text { ALD/ } \\
\text { VOC }\end{array}$ \\
\hline 3 & 2.09 & 5 & 7 & $\begin{array}{l}0800- \\
1100\end{array}$ & $\begin{array}{l}1,3,6,14 \\
22,24,29\end{array}$ & $30-54$ & Spring & VOC \\
\hline 4 & 0.32 & 5 & 7 & $\begin{array}{l}0900- \\
1200\end{array}$ & $\begin{array}{c}1,2,7,9,12 \\
14,21,28\end{array}$ & 21-44 & Winter & VOC \\
\hline
\end{tabular}

${ }^{\mathrm{a}}$ Ventilation rate.

${ }^{\mathrm{b}}$ Data not available for all periods on some days.

${ }^{\mathrm{c}} \mathrm{ALD}=$ aldehydes; $\mathrm{VOC}=$ volatile organic compounds. 
Table 2. Exposure-relevant emission factors $\left(\mu \mathrm{g} \mathrm{cig}^{-1}\right)$ for ETS compounds measured under steady-cycle conditions in a furnished $50-\mathrm{m}^{3}$ room. ${ }^{\mathrm{a}}$

\begin{tabular}{|c|c|c|c|c|c|c|}
\hline Compound $^{\mathrm{b}}$ & $\mathrm{CV}^{\mathrm{c}}$ & $2 \mathrm{~h}^{-1}$ & $0.6 \mathrm{~h}^{-1}$ & $0.3 \mathrm{~h}^{-1}$ & $\begin{array}{c}\text { Mean } \\
\text { ratio to } \\
\text { single-day } \\
\text { EREFs }\end{array}$ & $\begin{array}{c}\text { Mean } \\
\text { ratio to } \\
\text { initial } \\
\text { EREFs }\end{array}$ \\
\hline \multicolumn{7}{|c|}{ Gas-Phase Organics } \\
\hline Formaldehyde & $0.11^{\mathrm{f}}$ & 1310 & 950 & $\mathrm{~N} / \mathrm{A}$ & N/A & 1.37 \\
\hline Acetaldehyde & $0.18^{f}$ & 2480 & 2360 & N/A & N/A & 1.20 \\
\hline Acrolein & 0.30 & 400 & 610 & 610 & 1.19 & 0.93 \\
\hline 1,3-Butadiene & 0.20 & 400 & 520 & 510 & 1.45 & 1.07 \\
\hline Isoprene & 0.19 & 2810 & 2950 & 2750 & 1.20 & 1.03 \\
\hline Acrylonitrile & 0.11 & 250 & 180 & 210 & 1.16 & 1.07 \\
\hline Acetonitrile & 0.13 & 930 & 1080 & 1210 & 1.28 & 1.00 \\
\hline 2-Butanone & 0.14 & 540 & 300 & 350 & 1.29 & 0.98 \\
\hline Benzene & 0.12 & 590 & 430 & 430 & 1.12 & 1.12 \\
\hline Toluene & 0.09 & 1270 & 990 & 860 & 1.23 & 1.15 \\
\hline Ethylbenzene & 0.09 & 170 & 150 & 150 & 1.18 & 1.17 \\
\hline m,p-Xylene & 0.09 & 480 & $N / A^{g}$ & 420 & 1.20 & 1.15 \\
\hline o-Xylene & 0.10 & 91 & 77 & 91 & 1.20 & 1.28 \\
\hline Styrene & 0.18 & 210 & 170 & 300 & 1.45 & 1.22 \\
\hline 1,2,4-ТMB & 0.16 & 70 & 73 & 71 & 1.11 & 1.23 \\
\hline Naphthalene & 0.12 & 55 & 44 & 37 & 1.85 & 1.64 \\
\hline 2-Me-naphth & 0.14 & 32 & 28 & 23 & 2.18 & 1.84 \\
\hline 1-Me-naphth & 0.14 & 29 & 27 & 22 & 2.13 & 1.92 \\
\hline Phenol & 0.21 & 360 & 160 & 220 & 2.69 & 1.73 \\
\hline o-Cresol & 0.13 & 41 & 24 & 22 & 2.34 & 1.63 \\
\hline p-Cresol & 0.12 & 72 & 36 & 32 & 3.42 & 1.85 \\
\hline m-Cresol & 0.12 & 35 & 14 & 16 & 2.26 & 1.53 \\
\hline \multicolumn{7}{|c|}{ ETS-Specific Compounds } \\
\hline Pyridine & 0.11 & 530 & 390 & 380 & 1.29 & 1.20 \\
\hline 2-Picoline & 0.12 & 140 & 130 & 110 & 1.27 & 1.20 \\
\hline Pyrrole & 0.18 & 460 & 380 & 230 & 1.70 & 1.21 \\
\hline 3,4-Picoline ${ }^{\mathrm{h}}$ & 0.09 & 350 & 300 & 260 & 1.35 & 1.31 \\
\hline 3-EP & 0.14 & 640 & 530 & 490 & 1.57 & 1.45 \\
\hline Nicotine & 0.12 & 3070 & 1660 & 820 & 2.30 & 1.96 \\
\hline Myosmine & 0.18 & 160 & 92 & 60 & 2.12 & 1.83 \\
\hline
\end{tabular}

${ }^{a}$ Steady-cycle achieved after 2 weeks of smoking during the same 3-h period each day for 6-7 days per week. ${ }^{\mathrm{b}}$ 1,2,4-TMB = 1,2,4-trimethylbenzene; 2-me-naphth = 2-methylnaphthalene; 1-me-naphth = 1-methylnaphthalene; 3$\mathrm{EP}=3$-ethenylpyridine. ${ }^{\mathrm{c}}$ Coefficient of variation (CV) calculated in previous study (Singer et al., 2002) from the ratios of 8 pairs of replicate experiments. ${ }^{\mathrm{d}}$ Single-day EREFs from Singer et al. (2002). ${ }^{\text {e }}$ Initial EREFs calculated from first 2 sampling days, which occurred during the first 3 days of smoking. ${ }^{\mathrm{f}} \mathrm{CV}$ for formaldehyde and acetaldehyde calculated as mean of individual period CVs for each of 3 daily periods during experiments 1-2 ( $n=6$ ); individual period CVs calculated from measurements on 3 or more sampling days during each experiment. ${ }^{\mathrm{g}}$ Thermal desorber on inletting system contaminated with p-xylene during this experiment. ${ }^{\mathrm{h}}$ 3,4-Picoline previously identified as 3-picoline (Singer et al., 2002). 


\section{Figure Captions}

Figure 1. Time-resolved concentrations of toluene, 3-ethenypyridine and 2-

methylnaphthalene in a 50- $\mathrm{m}^{3}$ furnished room continuously ventilated at $0.3,0.6$

or $2.1 \mathrm{~h}^{-1}$. Cigarettes were smoked during a 3-h period each day (0-3 h), starting

on Day 1. Smoking occurred daily at 0.3 and $2.1 \mathrm{~h}^{-1}$ and $6 \mathrm{~d} \mathrm{wk}^{-1}$ at $0.6 \mathrm{~h}^{-1}$.

Figure 2. Progression of exposure relevant emission factors (EREFs) for benzene, naphthalene and nicotine. Smoking occurred daily at 0.3 and $2.1 \mathrm{~h}^{-1}$ and $6 \mathrm{~d} \mathrm{wk}^{-1}$ at $0.6 \mathrm{~h}^{-1}$.

Figure 3. Fraction of total potential ETS exposure $\left(\mu \mathrm{h} \mathrm{h}^{-3}\right)$ by daily period resulting from 3-h of smoking (0-3 h) in a 50- $\mathrm{m}^{3}$ furnished room. Calculated from average concentrations measured over multiple sampling days during weeks 3-4 of each experiment.

Figure 4. Potential indirect exposures to ETS organic vapors for simulated habitual smoking of 5 cig d $^{-1}$ in a $50-\mathrm{m}^{3}$ furnished room. Smoking occurred during hours 0-3 each day. Potential indirect exposures were calculated from measured concentrations during weeks 3-4 of each experiment. Measurements for the $0.6 \mathrm{~h}^{-1}$ experiment were divided by 2 to adjust from the actual smoking rate of 10 cig d $^{-1}$.

NOTE TO PUBLISHER: Figures are being provided by hard copy. 

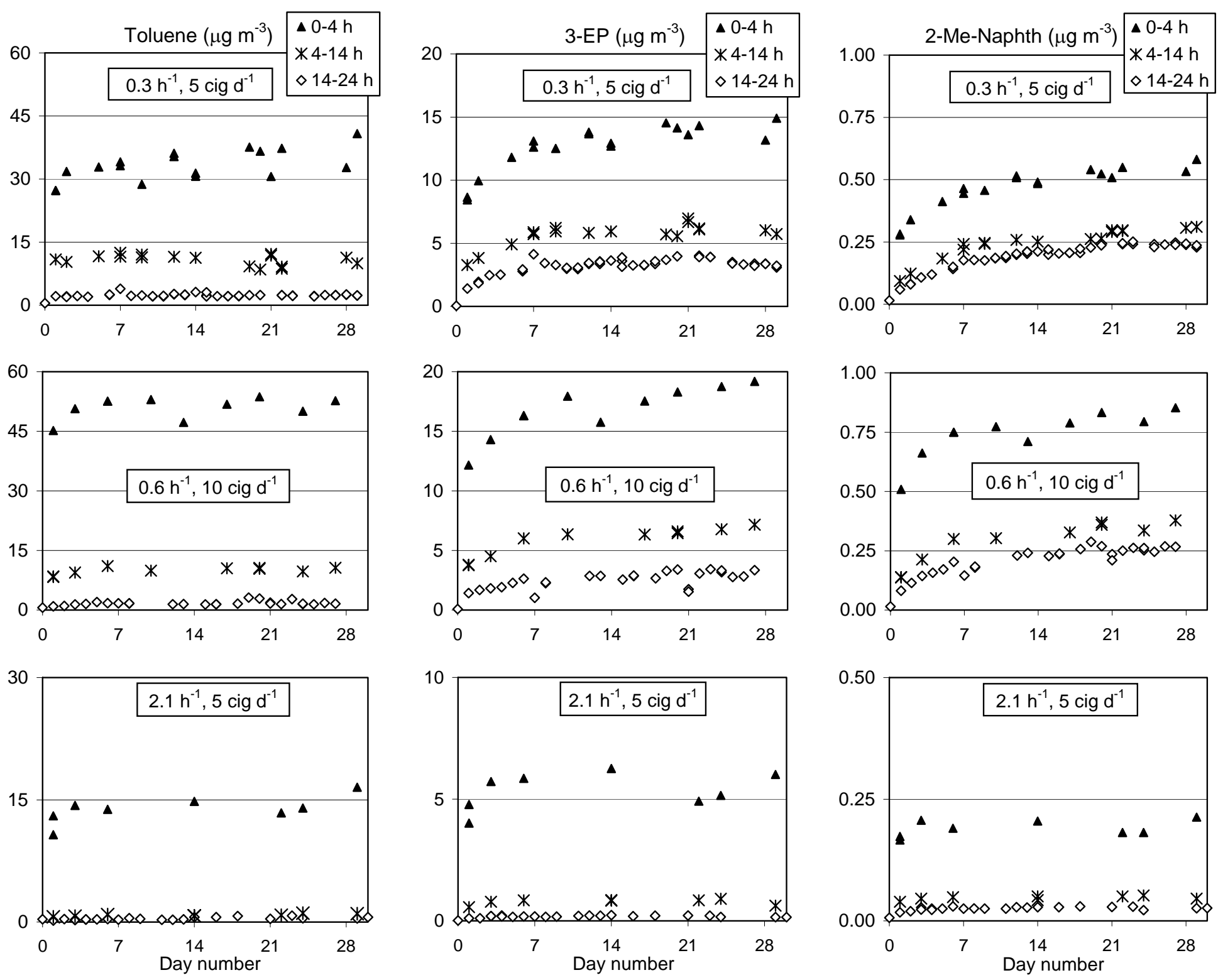

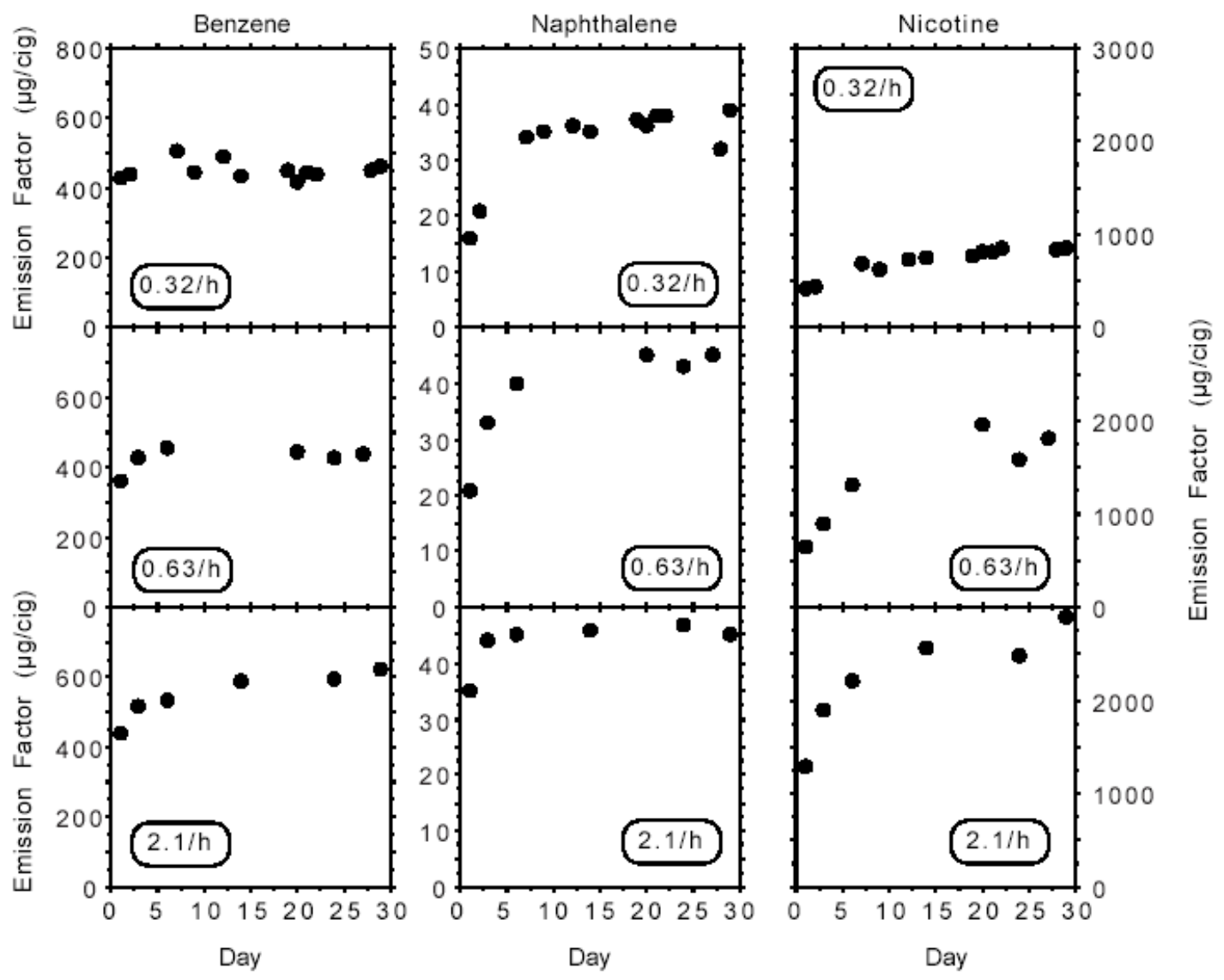


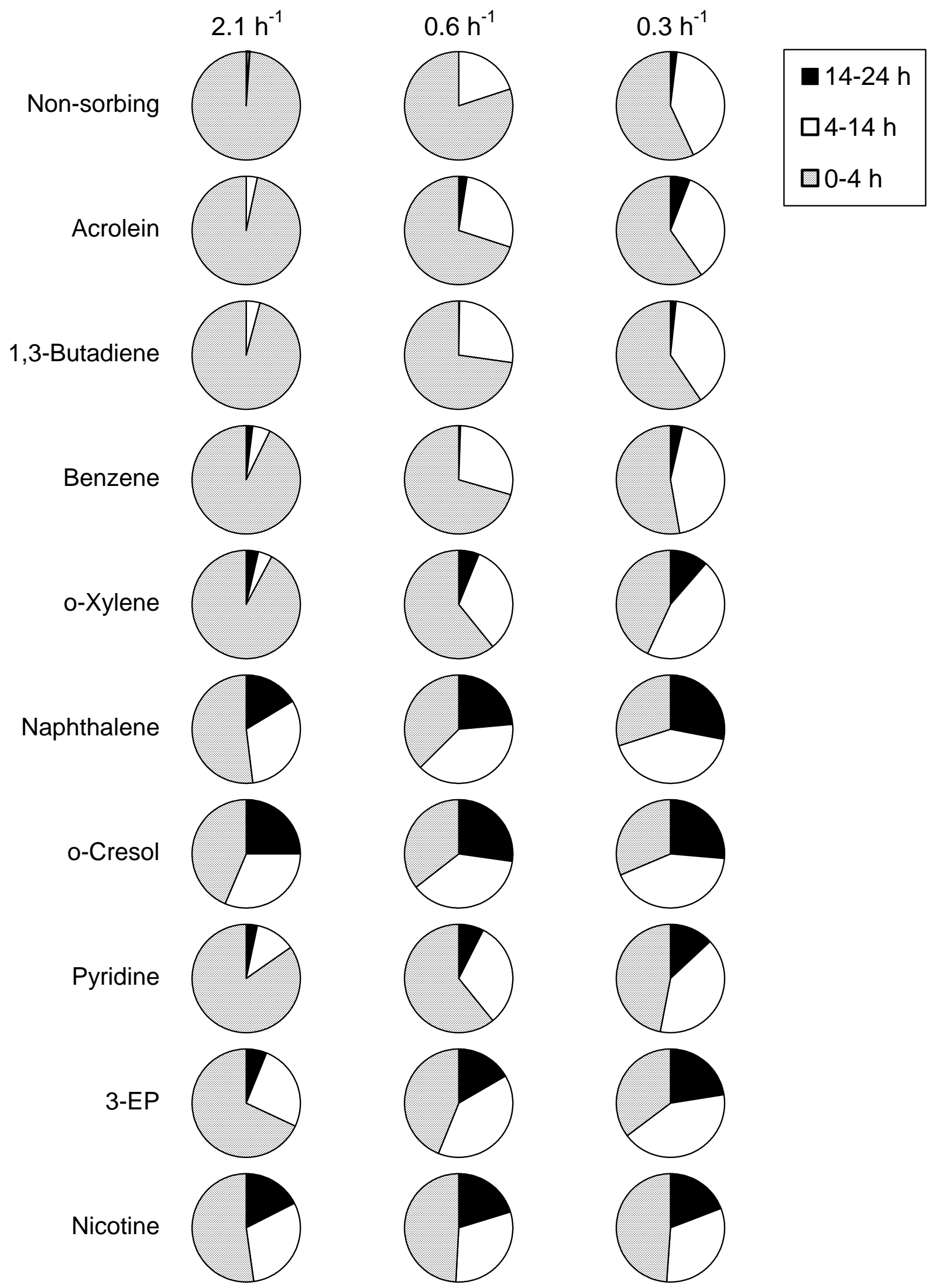




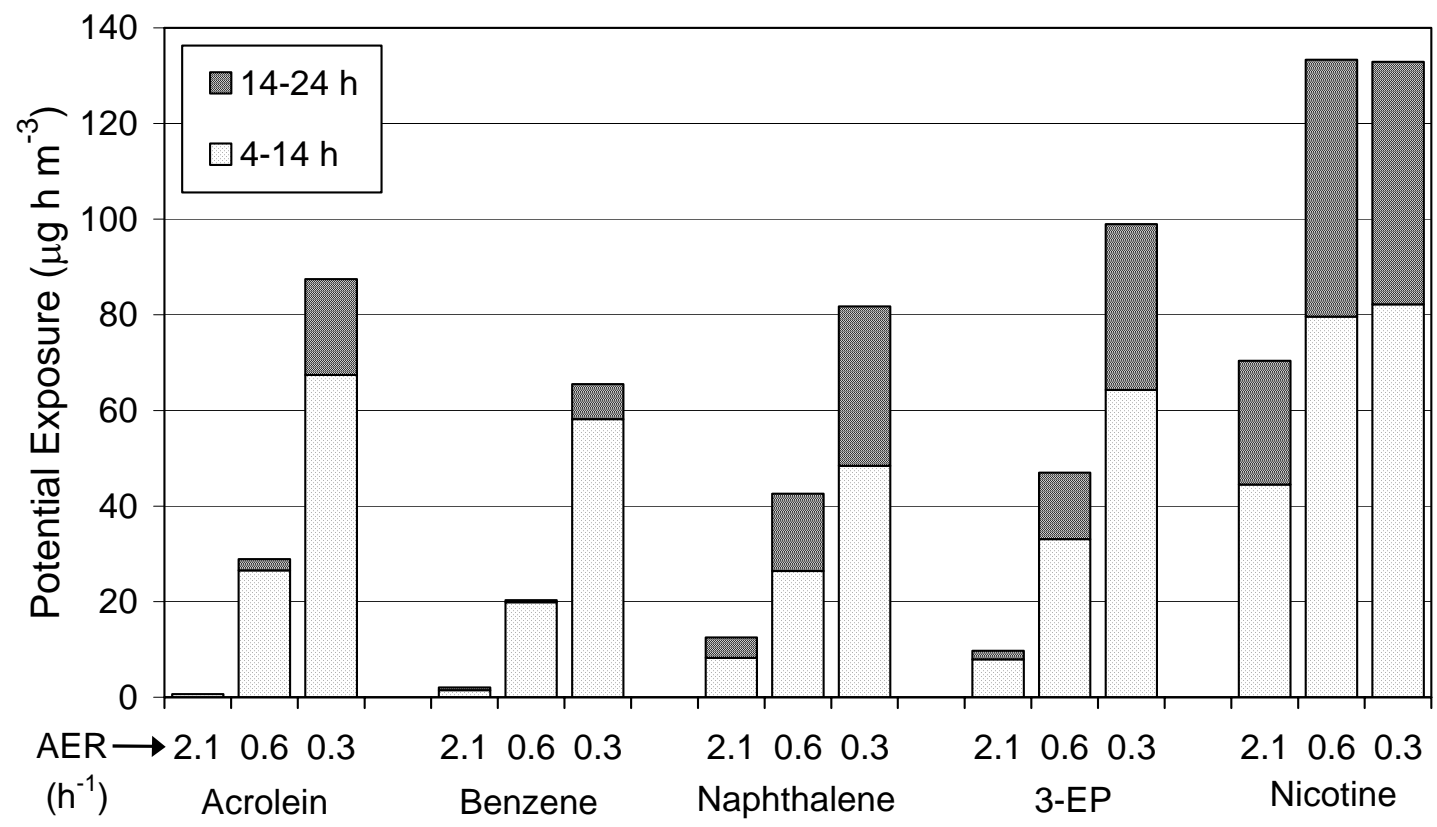

